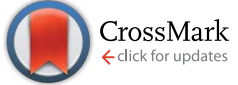

Cite this: RSC Adv., 2017, 7, 7519

\title{
The effect of selenium and polysaccharide of Atractylodes macrocephala Koidz. (PAMK) on endoplasmic reticulum stress and apoptosis in chicken spleen induced by heat stress
}

\begin{abstract}
Danning Xu, † Wanyan Li,† Bingxin Li, $\ddagger$ Yunbo Tianł* and Yunmao Huang $\ddagger^{*}$
Endoplasmic reticulum (ER) stress and oxidative stress are involved in different types of stress induced injuries. The aim of the present study was to evaluate the effect of selenium (Se), polysaccharide of Atractylodes macrocephala Koidz. (PAMK), and the combination of Se and PAMK on oxidative stress, ER stress and apoptosis in chicken spleen under heat stress (HS) condition. Chickens ( 1 day old, $n=200$ ) were randomly divided into two groups, the control (Con) group and the HS group. Then these chickens were treated with Se $\left(0.3 \mathrm{mg} \mathrm{kg}^{-1}\right)$, PAMK $\left(200 \mathrm{mg} \mathrm{kg}^{-1}\right)$ alone, and the combination of Se $\left(0.3 \mathrm{mg} \mathrm{kg}^{-1}\right)$ and PAMK (200 mg kg-1). The inducible NO synthase (iNOS)-NO activities (including NO content and iNOS activity), ER stress-related genes (GRP78, GRP94, ATF4, ATF6 and IRE) and apoptosis-related genes (caspase3 and $\mathrm{BCl}-2$ ) were examined by fluorescence quantitative PCR or western blot analysis. Apoptosis levels were also measured using ultrastructural observations. The results indicated that HS induced higher levels of NO content and the activity of iNOS which demonstrated that oxidative stress occurred in the chicken spleen. The ER stress-related genes (GRP78, GRP94, ATF4, ATF6 and IRE) and the apoptosis related gene caspase 3 were increased $(P<0.05)$, while $\mathrm{Bcl}-2$ was decreased $(P<0.05)$ induced by heat stress. These responses were alleviated by the treatment of Se, PAMK alone, and the combination of Se and PAMK $(P<0.05$ or not). The results showed that under common conditions, Se and PAMK could improve the immune response by reducing the levels of oxidative stress, ER stress and apoptosis to proper levels; however, under HS condition, Se and PAMK could change the abnormal levels of oxidative, ER damage and apoptosis to ameliorate the injury induced by HS. In addition, there existed a synergistic effect on the modulation of these biomarkers in chicken spleen between Se and PAMK. So both Se and PAMK may play important roles in regulating the immune function in chicken.
\end{abstract}

Received 4th December 2016 Accepted 24th December 2016

DOI: $10.1039 / \mathrm{c} 6 \mathrm{ra27730f}$

www.rsc.org/advances proapoptotic signal protein genes. ${ }^{6}$ Oxidative stress induced by HS has been reported as a primary factor that increases the pathogenesis of several diseases, decreases production results, and number of leucocytes in blood of chickens. ${ }^{7,8}$ Immune regulators alleviate immune system dysfunction and improve the condition of the host by regulating the immune response. Therefore, dietary manipulations are increasingly used as possible approaches to alleviate the harmful effects of high ambient temperature in poultry. ${ }^{9}$

Selenium (Se) is an essential trace element as it plays a crucial role in antioxidant defence mechanisms. ${ }^{10}$ Dietary Se can supply antioxidants and is particularly active in maintaining the production performance and breeding in poultry. ${ }^{\mathbf{1 1}} \mathrm{Se}$ also plays crucial protective effects in the immune organs. ${ }^{12} \mathrm{Se}$ supplementation has beneficial and detrimental effects on immunity to influenza vaccine in older adults. ${ }^{13}$ In addition, Se influences the antioxidation and immune function in heatstressed broilers. ${ }^{14}$ Therefore, Se is usually used as one immune regulator under HS condition in livestock production.
College of Animal Science \& Technology, Zhongkai University of Agriculture and Engineering, Guangzhou, 510225, China. E-mail: tyunbo@126.com; huangyunmao1111@163.com; Tel: +862089002081

$\dagger$ The authors contributed equally to this work.

\$ All other authors have read the manuscript and have agreed to submit this manuscript in its current form for consideration for publication Journal. 
Atractylodes macrocephala Koidz. (AMK) as a traditional Chinese herb medicine has been utilized for at least 2000 years. Polysaccharide of Atractylodes macrocephala Koidz. (PAMK) has been shown to have immune regulating functions, and has already widely used in the breeding industry in China. It is reported that PAMK can ameliorate metabolic status and improve immune function in early weaned pigs. ${ }^{15}$ However, as one newly identified immune regulator, the possible mechanism by which PAMK influences the immune function is unknown. In our previous studies, we found that the compound of PAMK and Se could alleviate the inflammatory response and regulate the expression of HSPs gene in chicken spleen which induced by heat stress. ${ }^{16,17}$

Both Se and PAMK preserve the roles of immune regulation, antioxidation, and or HS protective function. The effect of Se and PAMK on the regulation of immune function and injury under HS condition was less reported. In the current study, the objective was to investigate the effect of Se, PAMK, and the combination of Se and PAMK on NO content, iNOS activity, ER stress and apoptosis in a chicken immune system.

\section{Materials and methods}

\section{Chicken diet and sample collection}

Chickens were treated humanely, and the experiments received prior ethical approval in accordance with Zhongkai University of Agriculture and Engineering and under the approved protocol number SRM-11. Two hundred chickens were assigned randomly by body weight to one of eight dietary treatments. Each treatment was replicated five times with 5 chickens each. During the first 3 weeks of the experiment, the chambers were not separated and recommended brooding temperatures were applied, so that the temperature was reduced progressively from 33 to $23.9{ }^{\circ} \mathrm{C}$ by the end of the third week of age. The control diets were formulated with the Se $\left(1.5 \mathrm{mg} \mathrm{kg}^{-1} \mathrm{diet}\right)$ and PAMK $\left(0 \mathrm{mg} \mathrm{kg}{ }^{-1}\right)$. The Se supplemental diets were formulated with the Se (3 $\mathrm{mg} \mathrm{kg}^{-1}$ diet); the PAMK supplemental diets were formulated with the PAMK (200 $\mathrm{mg} \mathrm{kg}^{-1}$ ) and the combination group with Se (3 mg kg ${ }^{-1}$ diet) and PAMK (200 $\left.\mathrm{mg} \mathrm{kg} \mathrm{kg}^{-1}\right)$. Throughout the entire experimental period, the chickens were allowed ad libitum consumption of feed and water, feed intake and body weights were recorded weekly. Clinical symptoms and mortality were also recorded. At the end of the feeding experiment, chickens were killed with sodium pentobarbital. The spleen tissues were quickly removed, minced and stored at $-80{ }^{\circ} \mathrm{C}$ to determine the index of oxidative stress and isolate the RNA and protein. The remaining tissues were used in the preliminary experiment and served as standby tissues.

\section{Temperature and treatments}

After 3 weeks, at the recommended brooding temperatures, five cages per dietary treatment were subjected to either a high temperature or the optimum temperature. In one chamber, the ambient temperature was set to $37 \pm 1^{\circ} \mathrm{C}$. In the other chamber, the temperature was kept $23.9{ }^{\circ} \mathrm{C}$. The relative humidity was allowed to fluctuate, but not to levels below 55\%. For 4 weeks, the chickens were given the same dietary treatment. During the experiment, the chickens were on a lighting schedule of $24 \mathrm{~h}$ of light, with an average light intensity of $15 \mathrm{~lx}$.

\section{Ultrastructural observations}

For electron microscopy, spleen tissue specimens were fixed with $2.5 \%$ glutaraldehyde in $0.1 \mathrm{M}$ sodium phosphate buffer (pH 7.2) for $3 \mathrm{~h}$ at $4{ }^{\circ} \mathrm{C}$, washed in the same buffer for $1 \mathrm{~h}$ at $4{ }^{\circ} \mathrm{C}$ and postfixed with $1 \%$ osmium tetroxide in sodium phosphate buffer for $1 \mathrm{~h}$ at $4{ }^{\circ} \mathrm{C}$. The tissues were then dehydrated in a graded series of ethanol starting at $50 \%$ for $10 \mathrm{~min}$ after two changes in propylene oxide. The tissue specimens were embedded in araldite. Ultrathin sections were stained with Mguranyl acetate and lead citrate for transmission electron microscope evaluation.

\section{Determination of NO content and iNOS activity in spleen}

Chicken spleens were homogenized on ice in physiological saline and centrifuged at $700 \times g$ for $15 \mathrm{~min}$ at $4{ }^{\circ} \mathrm{C}$ before supernatant collection. Here, we detected NO and iNOS as indices of oxidative damage. The NO content and iNOS activity were determined using NO and iNOS activity assay kits (Nanjing Jiancheng Bioengineering Institute, Nanjing, China). The method used in the present study was according to the procedure previously published by Liu with an ELX800 Microplate reader (BioTek Instruments, USA) to detect the OD at 550 and $530 \mathrm{~nm}$, respectively. ${ }^{18}$

\section{Quantitative real-time PCR analysis}

The method of quantification used was followed by Yao. ${ }^{19}$ After quantification, the expression levels of autophagy genes were determined by quantitative reverse transcription PCR using SYBR Premix ExTaq TM (Takara, China) and an ABI PRISM 7500 real-time PCR system (Applied Biosystems). The PCR primers (Table 1) were designed using Oligo Primer Analysis software (version6.0) and synthesized by Invitrogen (Shanghai, China).

Table 1 Gene-specific primers used in the real-time quantitative reverse transcription $\mathrm{PCR}$

Gene Primer sequence

GRP78

Forward 5'-GAATCGGCTAACACCAGAGGA-3'

GRP94 Reverse $5^{\prime}$-CGCATAGCTCTCCAGCTCATT- $3^{\prime}$

Bcl-2 Forward 5'-CAAAGACATGCTGAGGCGAGT- $3^{\prime}$

Bcl-2

Caspase3 Reverse $5^{\prime}$-TCCACCTTTGCATCCAGGTCA-3'

ATF4 Forward 5'-ATCGTCGCCTTCTTCGAGTT-3' Reverse 5'-ATCCCATCCTCCGTTGTCCT- $3^{\prime}$ Forward 5'-CATCT GCATCC GTGCCTGA-3' Reverse $5^{\prime}$-CTCTCGG CTGTGGTGGTGAA- $3^{\prime}$ Forward $5^{\prime}$-GAATCGGCTAACACCAGAGGA- $3^{\prime}$ Reverse 5'-CGCATAGCTCTCCAGCTCATT-3'

ATF6 Forward $5^{\prime}$-CGTCGTCTGAACCACTTACTGA- $3^{\prime}$

IRE Reverse $5^{\prime}$-CCTTCTTTCCTAACAGCCACAC-3' Forward $5^{\prime}$-CTACAGGTCGCTCCTCACATC- $3^{\prime}$ Reverse $5^{\prime}$-ATCAGTCCTTCTGCTCCCATCT- $3^{\prime}$

GADPH Forward 5'-AGAACATCATCCCAGCGT- $3^{\prime}$ Reverse $5^{\prime}$-AGCCTTCACTACCCTCTTG- $3^{\prime}$ 
Reactions consisted of the following: $10 \mu \mathrm{l}$ of $2 \times$ SYBR Green I PCR Master Mix (TaKaRa, China), $0.4 \mu$ l of $50 \times$ ROX reference Dye II, $0.4 \mu \mathrm{l}$ of each primer $(10 \mu \mathrm{M}), 2 \mu \mathrm{l}$ of diluted cDNA, and $6.8 \mu \mathrm{l}$ of PCR-grade water. The PCR program for amplification of detect genes and GAPDH consisted of $95{ }^{\circ} \mathrm{C}$ for $30 \mathrm{~s}$ followed by 40 cycles at $95{ }^{\circ} \mathrm{C}$ for $15 \mathrm{~s}$ and $60{ }^{\circ} \mathrm{C}$ for $30 \mathrm{~s}$. The results (fold changes) were expressed as $2^{-\Delta \Delta C_{\mathrm{t}}}$.

\section{Western blot analysis}

Protein extracts were subjected to SDS-polyacrylamide gel electrophoresis under reducing conditions on $12 \%$ gels. Separated proteins were then transferred to nitrocellulose membranes using a tank transfer for $2 \mathrm{~h}$ at $200 \mathrm{~mA}$ in Trisglycine buffer containing $20 \%$ methanol. Membranes were blocked with $5 \%$ skim milk for 16-24 h and incubated overnight with diluted primary chicken antibodies against Bcl-2 (1:500, Santa Cruz Biotechnology, USA), caspase 3 (1:100, Santa Cruz Biotechnology, USA) and GRP78 (1:1000, polyclonal antibody produced by our lab) followed by a horseradish peroxidase (HRP)conjugated secondary antibody against goat (Bcl-2) or rabbit (caspase3 and GRP78) IgG (1:1000, Santa Cruz Biotechnology, USA). To verify equal loading of the samples, the membrane was incubated with a monoclonal b-actin antibody (1:1000, Santa Cruz Biotechnology, USA), followed by a HRP-conjugated goat anti-mouse IgG (1:1000) secondary antibody. The signal was detected by X-ray films (Trans Gen Biotech Co., China). The optical density (OD) of each band was determined using the Image VCD gel imaging system, and Bcl-2, caspase 3 and GRP78 expression were detected as the OD ratio between Bcl-2, caspase 3 and GRP78 and that of $\beta$-actin respectively.

\section{Statistical analysis}

Statistical analysis of all data was performed by using SPSS for Windows (version 13, SPSS Inc., Chicago, IL). When a main effect $(p<0.05)$ was identified by one-way analysis of variance, mean comparisons were followed. All data were checked for normal distribution and equal variance. Differences between means were assessed by Tukey's honestly significant difference tests. Data are expressed as mean \pm standard deviation. Differences were considered to be significant at $p<0.05$.

\section{Results}

\section{Ultrastructural changes}

No obvious ultrastructural changes were observed in all the control group (Fig. 1a and b). Under heat stress, the mitochondria from the spleen of HS group were vacuolated and the cristae had degenerated. The apoptotic cells showed typical condensed nuclei with horseshoe-like or crescent-shaped cytoplasmic organelles of an inconspicuous structure accompanied by nucleus shrinkage. The nuclei and organelles of some cells were unclear. The mitochondria were surrounded by fractured ER, and their rough ER were extended (Fig. 1c-e). When treating the HS chicken with Se or PAMK, the mitochondria damage was alleviated (Fig. $1 \mathrm{f}$ and $\mathrm{g}$ ). In addition, the combination of Se and PAMK further enhanced the solo effect of Se and PAMK (Fig. 1h).

\section{Changes in NO content and iNOS activity}

The effects of Se and PAMK on NO content and iNOS activity in spleen are shown in (Fig. 2). The results indicated that under common condition, there was no different in NO content and iNOS activity via Se treatment or PAMK $(p>0.05)$. But the combination of Se and PAMK could increase the activities of both NO and iNOS $(p<0.05)$. Under HS condition, the NO content and iNOS activity were decreased $(p<0.05)$. When treating the HS chicken with Se or PAMK, the NO content and iNOS activity were decreased $(p<0.05)$ compared with the HS group. In addition, the combination of Se and PAMK further enhanced the solo effect of Se and PAMK.

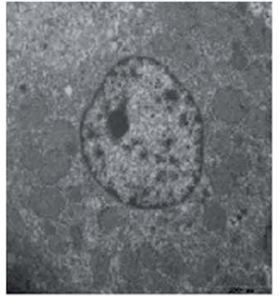

la control

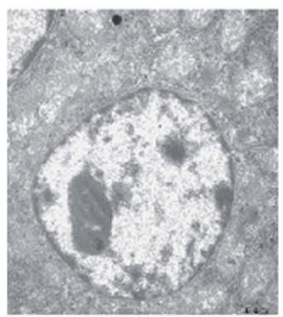

le HS

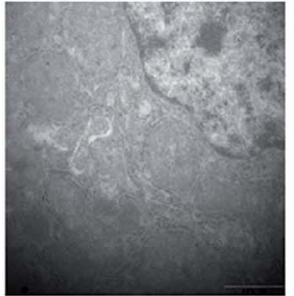

lb control

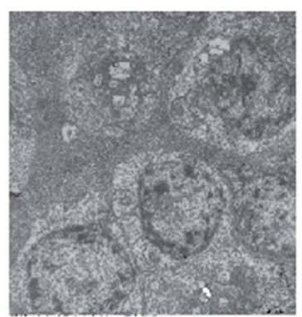

lf HS+PAMK

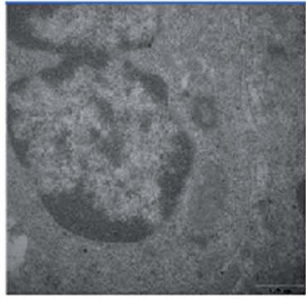

lc HS

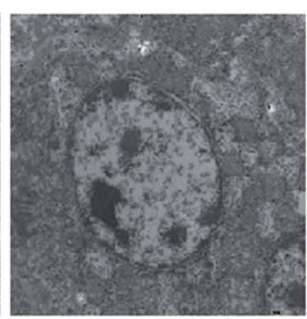

$\lg \mathrm{HS}+\mathrm{Se}$

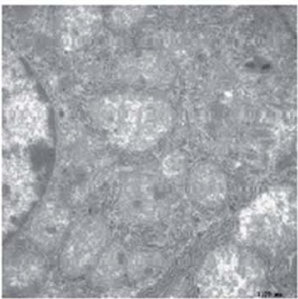

Id HS

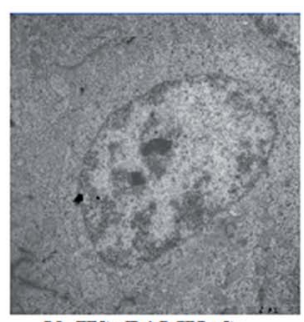

lh HS+PAMK+Se

Fig. 1 The ultrastructural changes in spleen of chickens in both control and heat stress group. 


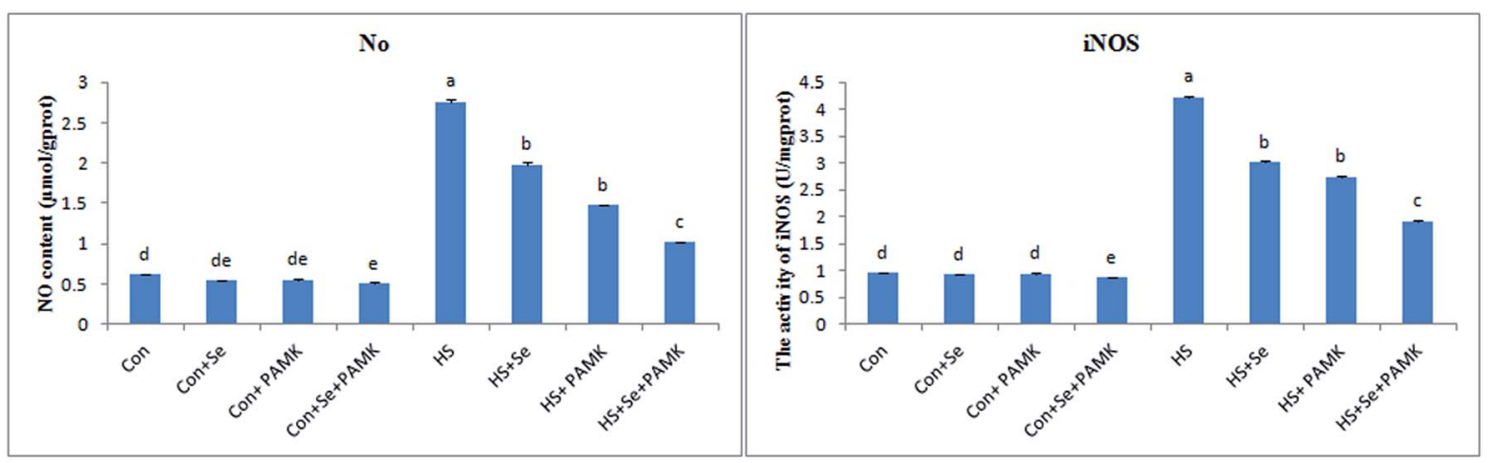

Fig. 2 The NO content and iNOS activity. Data are means $\pm \mathrm{SD}, n=5$. Bars without sharing a common letter were significantly different $(P<0.05)$.

Effects of Se and PAMK on the expression of ER stress-related genes (GRP78, GRP94, ATF4, ATF6 and IRE) in spleen of chicken which induced by heat stress

The effects of Se and PAMK on the expression of ER stressrelated genes in spleen are shown in (Fig. 3). The results indicated that under common condition, there was no different in mRNA level of GRP78, ATF4 and ATF6 gene via Se treatment or PAMK $(p<0.05)$, and Se treatment or PAMK could decrease the MRNA level of GRP94 and IRE genes. But the combination of Se and PAMK could decrease the mRNA level of all genes $(p<$
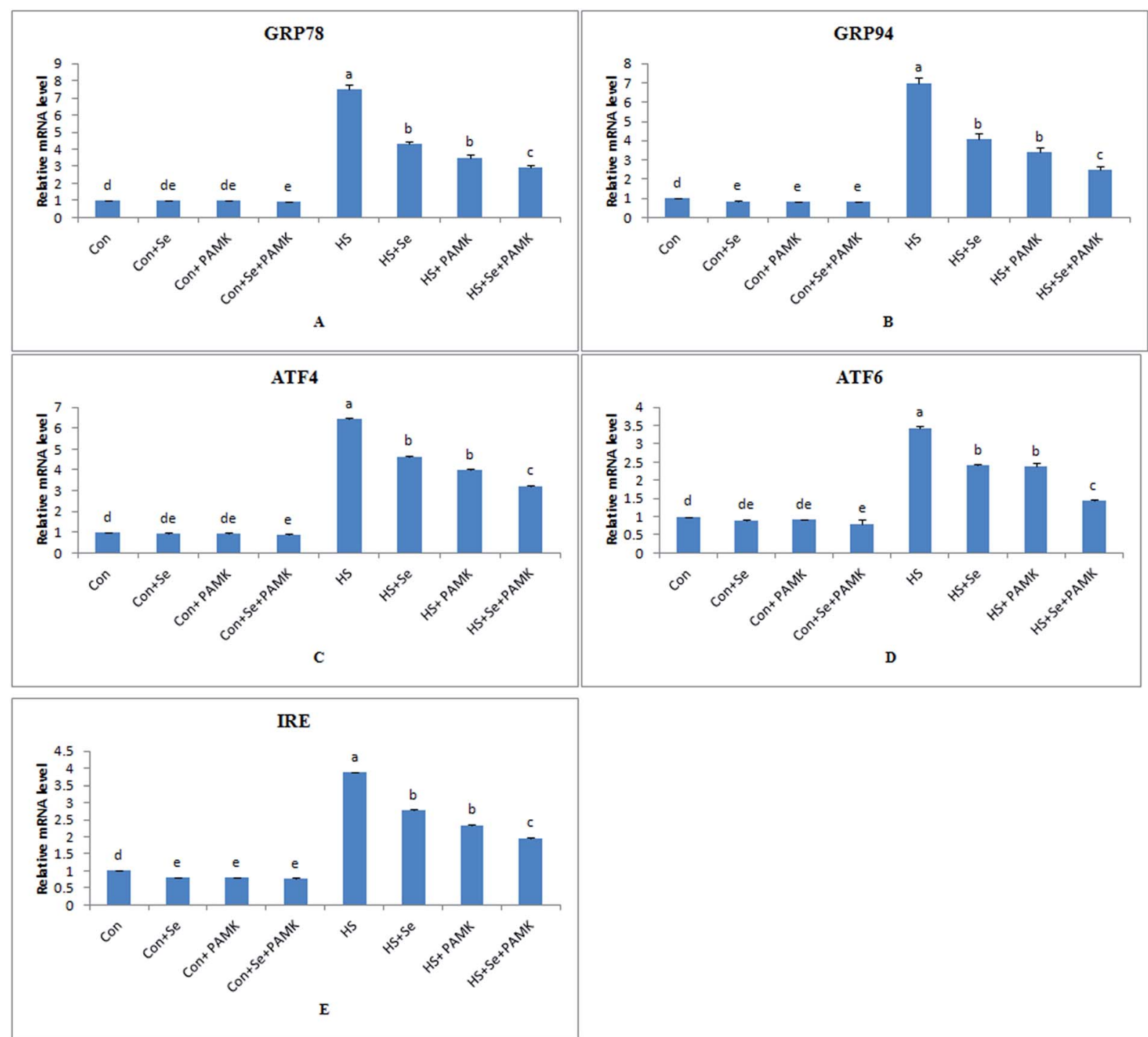

Fig. 3 The mRNA levels of ER stress-related genes (GRP78, GRP94, ATF4, ATF6 and IRE) in spleen of chicken. Data are means \pm SD, $n=5$. Bars without sharing a common letter were significantly different $(P<0.05)$. 
0.05). Under HS condition, the mRNA levels of all the genes were enhanced $(p<0.05)$. When treating the HS chicken with Se or PAMK, the mRNA level of all genes were decreased $(P<0.05)$ compared with the HS group. In addition, the combination of Se and PAMK further enhanced the solo effect of Se and PAMK.

\section{Effects of Se and PAMK on the expression of apoptosis-related genes (Bcl-2 and caspase3) in spleen of chicken which induced by heat stress}

The effects of Se and PAMK on the expression of apoptosisrelated genes in spleen are shown in (Fig. 4). The results showed that under common condition, Se treatment increased the mRNA level of Bcl-2 $(p<0.05)$, however, PAMK didn't cause any change in the mRNA level $(p<0.05)$. But the combination of Se and PAMK also could increase the mRNA of Bcl-2 gene $(p<$
0.05). The mRNA level of caspase 3 in each treated groups were not influenced $(p<0.05)$, except in the compound group. Under HS condition, the mRNA level of Bcl-2 was decreased $(p<0.05)$, but the mRNA level of caspase 3 was enhanced $(p<0.05)$. When treating the HS chicken with Se or PAMK, the mRNA level of Bcl2 was increased $(p<0.05)$, but the levels of caspase 3 was decreased $(p<0.05)$ compared with the HS group. In addition, the combination of Se and PAMK further enhanced the solo effect of Se and PAMK.

\section{Western bolt analysis of GRP78, Bcl-2 and caspase3 levels}

The protein expression levels of GRP78, Bcl-2 and caspase 3 were examined by western blots. The results (Fig. 5) showed that under common condition, there was no different in protein levels of GRP78 and caspase3 genes via Se treatment or PAMK ( $p$

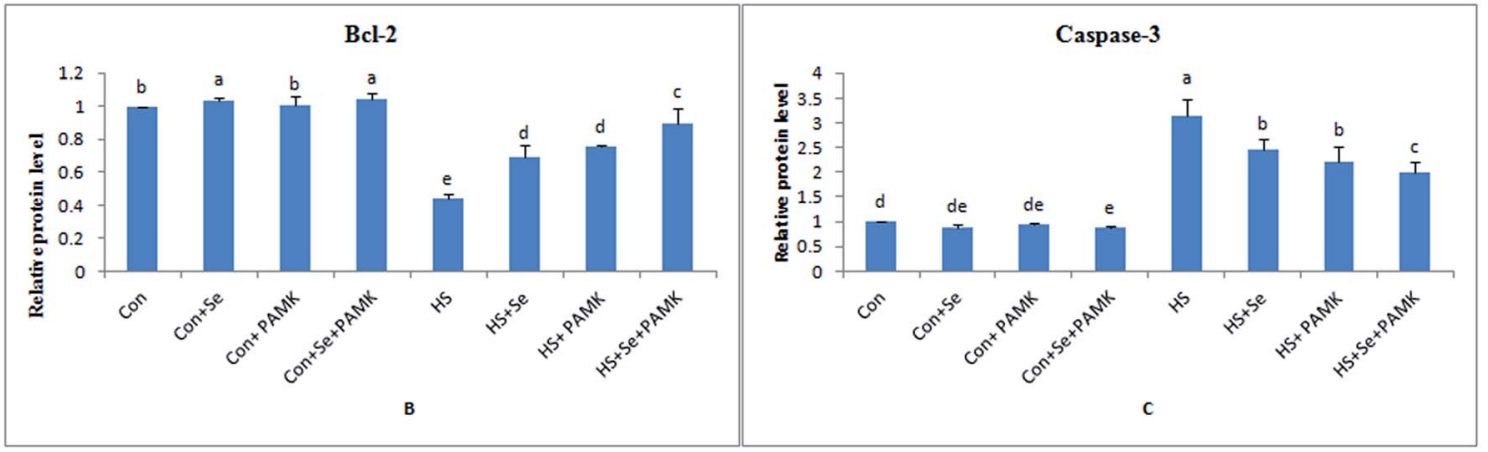

Fig. 4 The mRNA levels of apoptosis-related genes (Bcl-2 and caspase3) in spleen of chicken. Data are means $\pm S D, n=5$. Bars without sharing a common letter were significantly different $(P<0.05)$.

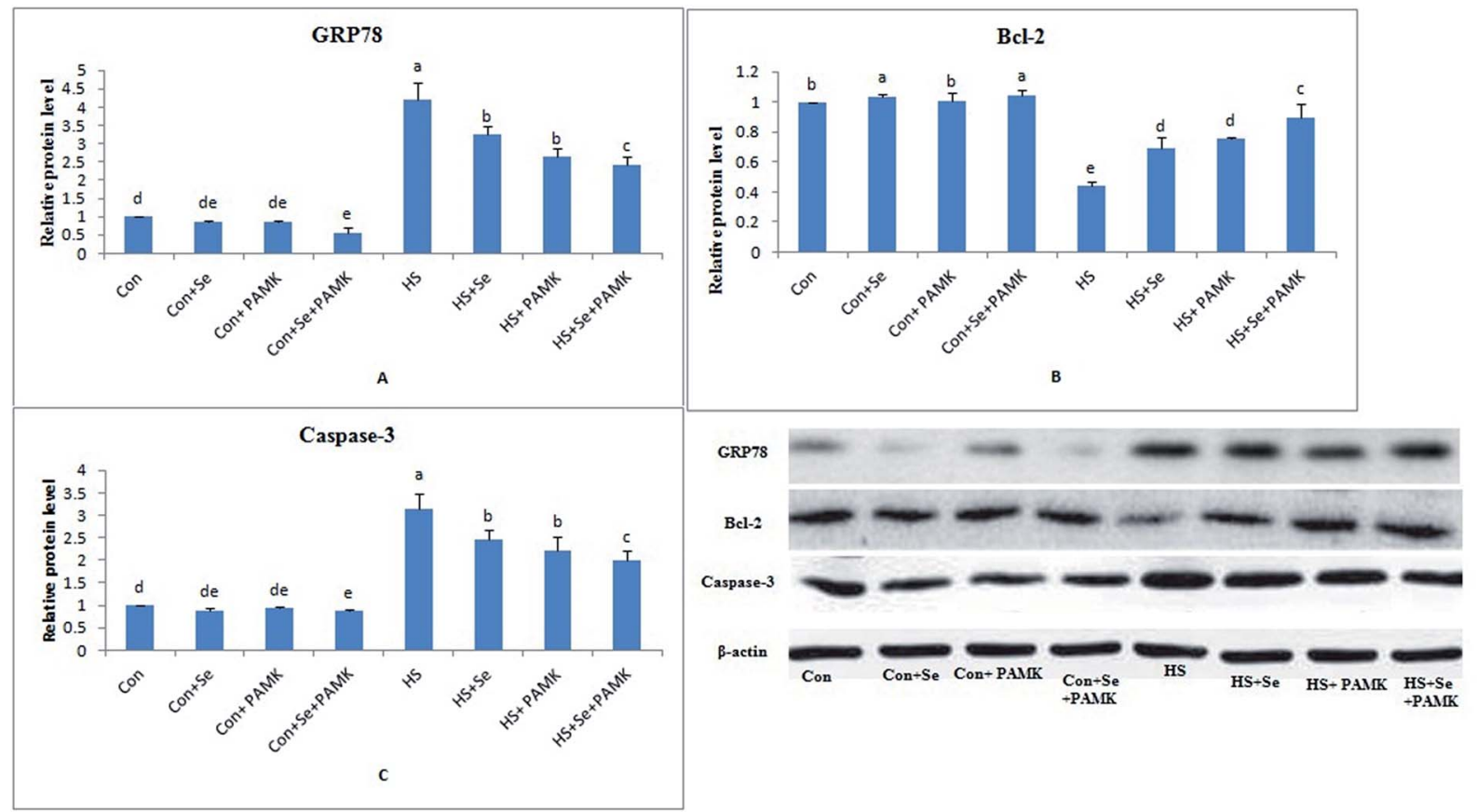

Fig. 5 The protein levels of GRP78, Bcl-2 and caspase3 as shown by images of western blot analyses, and the protein levels of GRP78, Bcl-2 and caspase 3 in spleen of chicken. Data are means $\pm \mathrm{SD}, n=5$. Bars without sharing a common letter were significantly different $(P<0.05)$. 
$<0.05$ ), however, Se treatment could increase the protein level of Bcl-2 gene. And the combination of Se and PAMK could decrease the protein level of GRP78 and caspase3 genes $(p<$ 0.05 ), increase the protein level of $\mathrm{Bcl}-2$ gene. Under $\mathrm{HS}$ condition, the protein levels of GRP78 and caspase 3 genes were enhanced $(p<0.05)$, and Bcl-2's protein level was reduced. When treating the HS chicken with Se or PAMK, the protein level of GRP7 8 and caspase 3 were decreased $(p<0.05)$ compared with the HS group, however the protein level of Bcl-2 was increased. Moreover, the results revealed that protein expression of the three genes was consistent with their mRNA responses to Se and PAMK in spleen of chicken which induced by heat stress.

\section{Discussion}

Environmental stress (ES) is an event that most animals experience and that induces a number of responses involving immune system. As one important target of ES, the morphology of immune organs was significantly influenced by cold stress and heat stress. The research of Wang et al. demonstrated that the morphologic damages in immune organs were occurred in chicken induced by cold stress. ${ }^{20}$ Similar to prior studies, our current study showed that heat stress induced mitochondria damage and apoptosis in chicken spleen.

NO has broad biological functions as an active intracellular messenger. The overproduction of NO contributes to numerous pathological processes. ${ }^{21}$ Several studies have demonstrated the importance of NOS-mediated signaling in many ES models. The research of Zhang et al. demonstrated that both acute and chronic cold stress may cause duodenum oxidative stress and change in iNOS, which was related to the intestinal damage process. ${ }^{22}$ The study of Slawinska et al. pointed out that the iNOS activity was up-regulated in chicken macrophage-like cell line which induced by heat stress environment. ${ }^{23}$ In the current study, an increased NO content and iNOS activity were observed in the spleen of chicken which induced by heat stress compared with the common condition. These results indicated that under conditions of heat stress, the antioxidant defence system was undermined in vivo, which led to the accumulation of ROS and the subsequent release of a large number of inflammatory mediators while stimulating an increase in iNOS expression and the excessive release of NO. ${ }^{24}$

The occurrence of oxidative stress induced continuous ER stress through interfering with the oxidation of the internal environment of the ER. Yao's research showed that the ER stress response may be downstream of oxidative stress. When ER stress occurred, chaperone proteins, are recruited to support the folding of new proteins. ${ }^{25}$ And the UPR also can enhance the capabilities of the ER to process paraproteins and up-regulate the expression of ER chaperones GRP78 and GRP94. ${ }^{26} \mathrm{~A}$ previous study showed that the expression of ER stressassociated signaling molecules including GRP78, GRP94 and ATF4 were increased induced by the pyrrolidine dithiocarbamate/Cu complex. ${ }^{27}$ In our study, the expression GRP78 and GRP94 were significantly elevated in the spleen of chicken induced by heat stress. The up-regulated expression of these proteins clearly indicated that heat stress could lead to ER stress. In addition, ER lesions were observed by ultrastructural observations in chicken spleen under heat stress condition. In addition, several pathways including IRE1, PERK, and ATF6 were also relate to ER stress. Among them, the PERK pathway enhanced translation of mRNAs, including ATF4. ${ }^{28}$ When ER stress occurred, the mRNA levels of those gene were increased. ${ }^{29,30}$

Caspases are key regulators of cell apoptosis, and are involved in ER stressed-induced apoptosis. ${ }^{31}$ When exposed to glucose-induced apoptosis, caspase 3 activity was significantly increased in treated PC12 cells compared to controls. ${ }^{32}$ Moreover, during the process of apoptosis induced by ER stress, the Bcl-2 mRNA and protein expressions were decreased in lung epithelial cells. ${ }^{27}$ In the current study, both caspase 3 mRNA and protein increased and Bcl-2 mRNA and protein levels decreased in the heat stress group. In addition, apoptosis was observed by ultrastructural observations in chicken spleen. These results showed that ER stress was really related to the occurrence of apoptosis.

Se is an famous antioxidant and plays an important role in immunological functions in humans and many other species. ${ }^{33}$ Previous studies showed that adequate Se level played an important role in nitric oxide mediated apoptosis induced by cadmium in chicken immune organs. ${ }^{15,34}$ Additionally, Se influenced the antioxidative and immune functions in HS broilers. ${ }^{14}$ In the present research, Se could improve the immune functions including oxidative damage, endoplasmic reticulum stress and apoptosis in HS-treated broilers.

Several nutritional approaches such as supplementation of diets with phytochemicals have been used attempted to attenuate the negative effects of heat stress. ${ }^{35}$ Diets supplemented with tomato pomace could affect the growth, immune, and antioxidant responses of heat stress-exposed broilers. ${ }^{36}$ Dietary supplementation of either betaine or turmeric rhizome powder alone or in combination could partially ameliorate some of the detrimental effects of HS in broilers. ${ }^{37}$ Lemon verbena and vitamin $\mathrm{C}$ improved some performance data and blood metabolites which somehow suppressed the negative effects of heat stress. ${ }^{38}$ In our current research, add PAMK also could improve the immune functions, it showed that PAMK had the similar function as the above phytochemicals.

In summary, the present study showed that HS induced the occurrence of oxidative damage, endoplasmic reticulum stress and apoptosis in chicken spleen which affects the immune function.

Both Se and PAMK could serve as the immune regulator under HS condition. And there also existed synergistic effect between Se and PAMK, which may enhance the ability of Se and PAMK to improve immune function.

\section{Acknowledgements}

This work was supported by Training Program for Outstanding Young Teachers in Higher Education of Guangdong Province (No. Yq2013098), National Natural Science Foundation of China (No. 31402162), Waterfowl Healthy Breeding Engineering 
Research Center of Guangdong Higher Education Institutes (No. GCZX-A1307), China Spark Program (2015GA780003), Science \& Technology Planning Project of Guangdong Province (2015B020202012), Modern Agriculture Industry Technology System in Guangdong (2016LM1113).

\section{References}

1 K. Sahin, N. Sahin, S. Yaralioglu and M. Onderci, Biol. Trace Elem. Res., 2002, 85, 59-70.

2 H. Akhavan-Salamat and H. Ali Ghasemi, Trop. Anim. Health Prod., 2016, 48, 181-188.

3 M. M. Mashaly, G. L. Hendricks 3rd and M. A. Kalama, Poult. Sci., 2004, 83, 889-894.

4 J. L. Morrow-Tesch, J. J. McGlone and J. L. Salak-Johnson, J. Anim. Sci., 1994, 72, 2599-2609.

5 R. L. Starkie, M. Hargreaves, J. Rolland and M. A. Febbraio, Brain, Behav., Immun., 2005, 19, 404-412.

6 H. D. Yao, Q. Wu, Z. W. Zhang, J. L. Zhang, S. Li, J. Q. Huang, F. Z. Ren, S. W. Xu, X. L. Wang and X. G. Lei, J. Nutr., 2013, 143(5), 613-619.

7 C. Huang, H. Jiao, Z. Song, J. Zhao, X. Wang and H. Lin, J. Anim. Sci., 2015, 93, 2144-2153.

8 D. B. Nathan, E. D. Heller and M. Perek, Br. Poult. Sci., 1976, 17, 481-485.

9 R. U. Khan, S. Naz, Z. Nikousefat, M. Selvaggi, V. Laudadio and V. Tufarelli, World's Poult. Sci. J., 2012, 68, 477-490.

10 H. D. Yao, Q. Wu, Z. W. Zhang, S. Li, X. L. Wang, X. G. Lei and S. W. Xu, Biochim. Biophys. Acta, 2013, 4, 3112-3120.

11 Y. Wang, X. Zhan, D. Yuan, X. Zhang and R. Wu, Biol. Trace Elem. Res., 2011, 143, 1497-1507.

12 Z. W. Zhang, J. L. Zhang and Y. H. Gao, BioMetals, 2013, 26, 355-365.

13 K. Ivory, E. Prieto, C. Spinks, C. N. Armah, A. J. Goldson, J. R. Dainty and C. Nicoletti, Clin. Nutr., 2015, 15, 0034000344.

14 X. Liao, L. Lu, S. Li, S. Liu, L. Zhang, G. Wang, A. Li and X. Luo, Biol. Trace Elem. Res., 2012, 150, 158-165.

15 L. L. Li, F. G. Yin, B. Zhang, N. S. Zhu, L. I. Ai-Ke and F. H. Zhang, Livest. Sci., 2011, 142, 33-41.

16 D. Xu, W. Li, Y. Huang, J. He and Y. Tian, Biol. Trace Elem. Res., 2014, 160, 232-237.

17 D. Xu and Y. Tian, Biol. Trace Elem. Res., 2015, 168, 235-241.
18 C. Liu, J. Fu, C. Liu and S. Li, RSC Adv., 2015, 5, 50549-50556. 19 H. Yao, R. Fan, X. Zhao, W. Zhao, W. Liu, J. Yang, H. Sattar, J. Zhao, Z. Zhang and S. Xu, Oncotarget, 2016, 7(36), 5761857632.

20 J. T. Wang, S. Li, J. L. Li, J. W. Zhang and S. W. Xu, Poult. Sci., 2009, 8, 2549-2554.

21 B. Rey, D. Roussel, L. Teulier, P. Eyenga, C. Degletagne, M. Belouze and C. Duchamp, FEBS Lett., 2011, 585, 173-177.

22 Z. W. Zhang, Z. H. Lv, J. L. Li, S. Li, S. W. Xu and X. L. Wang, Poult. Sci., 2011, 7, 1555-1561.

23 A. Slawinska, J. C. Hsieh, C. J. Schmidt and S. J. Lamont, PLoS One, 2016, 11(10), e0164575.

24 J. Yu, H. Yao, X. Gao, Z. Zhang, J. F. Wang and S. W. Xu, Biol. Trace Elem. Res., 2015, 163, 144-153.

25 G. S. Hotamisligil, Cell, 2010, 140, 900-917.

26 A. S. Lee, Methods, 2005, 35, 373-381.

27 Y. W. Chen, K. L. Chen, C. H. Chen, H. C. Wu, C. C. Su, C. C. Wu, T. D. Way, D. Z. Hung, C. C. Yen, Y. T. Yang and T. H. Lu, Toxicol. Lett., 2010, 199, 333-340.

28 J. L. Armstrong, R. Flockhart, G. J. Veal, P. E. Lovat and C. P. Redfern, J. Biol. Chem., 2010, 285, 6091-6100.

29 Y. A. Seong, P. G. Shin and G. D. Kim, Internet J. Oncol., 2013, 42, 1045-1051.

30 Y. A. Seong, P. G. Shin, J. S. Yoon, A. K. Yadunandam and G. D. Kim, Cell Biochem. Biophys., 2014, 68, 369-377.

31 T. J. Fan, L. H. Han, R. S. Cong and J. Liang, Acta Biochim. Biophys. Sin., 2005, 37, 719-727.

32 A. M. Sharifi, H. Eslami, B. Larijani and J. Davoodi, Neurosci. Lett., 2009, 459, 47-51.

33 Z. Zhang, M. Bi, Q. Liu, J. Yang and S. Xu, Oncotarget, 2016, 7(47), 77110-77116.

34 Z. W. Zhang, J. L. Zhang, Y. H. Gao, Q. H. Wang, S. Li, X. L. Wang and S. W. Xu, BioMetals, 2013, 26, 355-365.

35 A. H. Perai, H. Kermanshahi, H. Nassiri Moghaddam and A. Zarban, Int. J. Biometeorol., 2015, 59, 453-462.

36 S. J. Hosseini-Vashan, A. Golian and A. Yaghobfar, Int. J. Biometeorol., 2016, 60, 1183-1192.

37 H. Akhavan-Salamat and H. A. Ghasemi, Trop. Anim. Health Prod., 2016, 48, 181-188.

38 F. Rafiee, M. Mazhari, M. Ghoreishi and O. Esmaeilipour, J. Anim. Physiol. Anim. Nutr., 2016, 100, 807-812. 